\title{
A systematic review of the effectiveness of clinical nurse specialist interventions in patients with chronic obstructive pulmonary disease (COPD)
}

Original article

\author{
Hua Yin ${ }^{a, *}$, Ling Yang ${ }^{b}$, Qiao Ye ${ }^{b}$
}

aDepartment of Respiratory Medicine, Zhuhai People's Hospital (The Third Hospital Affiliated to Jinan University), Zhuhai, Guangdong 519000, China ${ }^{b}$ Nursing Department, Zhuhai People's Hospital (The Third Hospital Affiliated to Jinan University), Zhuhai, Guangdong 519000, China

\section{Received: 3 September 2017; Accepted: 25 November 2017; Published: 20 June 2018}

\begin{abstract}
Objective: This review aimed to examine the effectiveness of clinical nurse specialist (CNS) interventions in patients with chronic obstructive pulmonary disease (COPD). COPD significantly affects people's health worldwide. With the development in nursing, CNSs are playing increasingly important roles in different departments. However, the studies on the effectiveness of CNSs in COPD are not as well organized as the studies on the effectiveness of CNSs in bronchiectasis and asthma. Therefore, this review aims to find some updated evidence on the CNS interventions for patients with COPD and on whether these interventions are effective. Methods: A narrative analysis of the data was performed for the eligible studies. Four databases were chosen: CINAHL, MEDLINE, British Nursing Index, and Cochrane Library. Other websites such as the National Institute for Health and Clinical Excellence, National Health Service Evidence, Association of Respiratory Nurse Specialists, and National Association of Clinical Nurse Specialist were searched as well. Two reviewers performed study identification independently, and all the retrieved articles were stored using the EndNote X7 software. The risk of bias in the included studies was assessed using the Cochrane Collaboration's risk of bias tool. Results: A total of nine studies were included in this review. There were five current interventions by CNSs for patients with COPD. These interventions were home nursing support, CNS's supported discharge, multidisciplinary cooperation programs, nurse-led care programs, and self-care management education. The effectiveness of these five interventions was evaluated individually. There is lowto moderate-quality evidence indicating that home nursing support interventions may have a positive effect on mortality and quality of life. No significant difference in quality of life has been found between the CNS-supported discharge intervention and the usual service. The multidisciplinary cooperation program probably had a positive effect on quality of life in patients with COPD. Both nurse-led care and self-care management education intervention had a positive effect on mortality of patients with COPD.

Conclusions: The findings of this review provide updated evidence on the effectiveness of CNS interventions for patients with COPD. Although nine trials were included and five types of interventions were identified, there is still lack of high-quality evidence.
\end{abstract}

Keywords: chronic obstructive pulmonary disease (COPD) • clinical nurse specialists (CNSs) • effectiveness $\bullet$ systematic review $\bullet$ advanced practice nurse $\bullet$ RNS • CNS led

(c) Shanxi Medical Periodical Press.

\section{Introduction}

Chronic obstructive pulmonary disease (COPD) is the fourth most common cause of death worldwide; more than three million people died of COPD in 2012. ${ }^{1}$ Risk factors of COPD include exposure to

*E-mail: huayin0408@163.com smoke, occupational dust, or fumes; recurrent respiratory infections; low socioeconomic status; poor nutrition; and asthma. ${ }^{2}$ COPD has a significant impact on individuals' health and frequently involves numerous complications, including hip fracture, male hypogonadism, sarcopenia, osteoporosis, cognitive impairment, malnutrition, and decreased awareness of hypoxia. ${ }^{3}$ 
Hence, individuals with COPD have high mortality, disability, and mortality.

Clinical nurse specialists (CNSs) play an important role in many aspects of health care including the management of COPD. Previous studies indicated that CNSs are associated with improvements in the health status of patients and their family satisfaction. ${ }^{4}$ In recent years, increasing evidence has shown the effectiveness of interventions led by CNSs in respiratory medicine. According to Rafferty and Elborn, CNSs in respiratory medicine are respiratory nurse specialists (RNSs). ${ }^{5}$ RNSs play a unique role and are making clinical decisions autonomously in the care and medical management of respiratory patients, such as in the facilities of palliative care, home-based care, hospital at home, and nurse-led clinics.

There are two existing reviews examining the effectiveness of nurse-led care in the management of bronchiectasis and asthma ${ }^{6,7}$. Both reviews reported positive impacts of CNS interventions on the management of respiratory disease. Yet, there is a lack of systematic reviews focusing on evaluating the effectiveness of interventions led by CNSs in the management of patients with COPD.

\section{Aims}

This review aimed to examine the effectiveness of CNS interventions in patients with COPD.

\section{Methods}

\subsection{Data sources and search terms}

Four databases were chosen: CINAHL, MEDLINE, British Nursing Index, and Cochrane Library. Besides these four databases, several websites were searched as well. Since the objective of this review was to explore specialist nurses' intervention for patients with COPD, the National Institute for Health and Clinical Excellence, National Health Service Evidence, Association of Respiratory Nurse Specialists, and National Association of Clinical Nurse Specialist websites were searched. Search terms included "COPD," "nurse specialist," "CNS," and "RNS." During the searching process, alternative keywords and search strategies were used as shown in Table 1.

\subsection{Types of study}

This review aimed to examine the effectiveness of interventions by CNSs. A randomized controlled trial (RCT), which is in the top position of the evidence hierarchy, is qualified and suitable to answer this kind of question. ${ }^{8}$ Therefore, only RCTs were included in this review.

\subsection{Inclusion and exclusion criteria}

Patients with COPD should be the only or at least the majority of participants. Both stable and exacerbation periods were included. Interventions were mainly or partly provided by CNSs or respiratory nurses playing an equivalent role as CNSs. CNSs in this review are nurses who have been trained and acquainted with specific knowledge in respiratory clinical practice, providing professional nursing care in hospitals, health facilities, community, or patients' homes. The comparison criteria were other interventions or interventions by other staff-for example, by physicians. Various outcomes were included and discussed. There was no special limitation about outcomes in the criteria.

\begin{tabular}{|c|c|c|c|c|c|}
\hline \multicolumn{2}{|c|}{ Population } & \multicolumn{2}{|c|}{ Interventions } & \multicolumn{2}{|c|}{ Outcome } \\
\hline & Chronic respiratory disease & 5. & Nurse specialist & 15. & Safe* \\
\hline 2. & Discharged & 6. & Clinical nurse specialist & 16. & Effective \\
\hline 3. & Chronic obstructive pulmonary disease & 7. & Advance practice nurse & 17. & Effectiveness \\
\hline \multirow{8}{*}{\multicolumn{2}{|c|}{ 4. COPD }} & 8. & Special nurse-led & 18. & Exercise tolerance \\
\hline & & 9. & Specialist-led & 19. & Pulmonary function \\
\hline & & 10. & RNS-led & 20. & Mortality \\
\hline & & 11. & RNS & 21. & Satisfaction \\
\hline & & 12. & CNS-led & 22. & The quality of life \\
\hline & & 13. & Home-based & 23. & Low cost \\
\hline & & 14. & Outreach nursing & 24. & Economic \\
\hline & & & & 25. & Cost effectiveness \\
\hline 26. & Combination of 1-4 using "OR" & 27. & Combination of 5-14 using "OR" & 28. & Combination of 15-25 using "OR" \\
\hline The & last step is to combine $26+27+28$ using "AND" & & & & \\
\hline
\end{tabular}

Table 1. Alternative keywords and search strategy. 
Exclusion criteria were studies in which the focus population was patients not clearly identified with COPD. The studies in which the interventions were simply performed by physicians were excluded. Owing to the language limitation of the authors, papers not in English or Chinese were excluded, and researchers compared before or after, without a control group, or not using an RCT were excluded.

\section{Results}

A total of nine studies were included in this review, and the search diagram is shown in Figure 1. The characteristics of the included studies are listed in Table 2.

\section{Methodological quality}

The Cochrane Collaboration's risk of bias tool was applied to assess risk of bias for included trials, and the results are shown in Figure 2. All nine included studies had a low risk of bias.

\section{Effectiveness of interventions}

Five types of interventions were identified from these included studies: home nursing support, ${ }^{9-11}$ CNS's

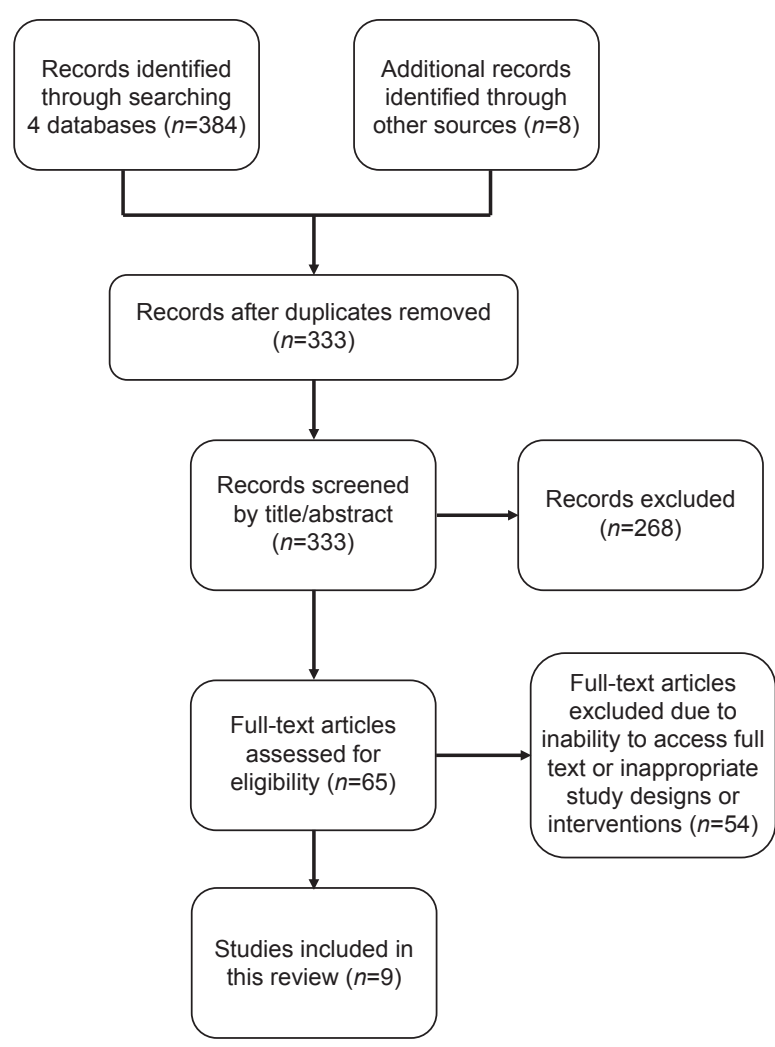

Figure 1. Article search diagram. supported discharge, ${ }^{12,13}$ multidisciplinary cooperation program, ${ }^{14}$ nurse-led care for patients with COPD,,${ }^{15-16}$ and self-care management education. ${ }^{15,17}$ According to different outcomes, the effectiveness of this investigation from the five intervention groups was presented as follows.

\subsection{Home nursing support}

Careful examination of the three studies in the home nursing support intervention ${ }^{9-11}$ reveals that CNSs implemented their home nursing support interventions mainly by visiting patients with COPD in their homes after discharge, giving them inspections, and giving health guidance and necessary advice. After interventions, outcomes of the utilization of hospital services may be equivalent to or better than the usual service. The quality of life was probably improved as all three RCTs support this improvement. At the same time, the mortality of patients was probably the same as the usual service according to the included studies. Drawing a conclusion about utilization of hospital services and lung function with this intervention is difficult as more evidence is needed.

Some similar studies on this topic also support this result. Lee et al. conducted a study on home nursing support in Hong Kong by community nursing visits. ${ }^{18}$ They found that, through the intervention, the psychological well-being of patients with COPD was significantly enhanced. Smith et al. also conducted a systematic review of the home care by outreach nursing for COPD. ${ }^{19}$ The conclusion of the review also shows that patients' quality of life was improved after intervention. Wong et al. developed a systematic review focused on outreach nursing programs for patients with COPD. ${ }^{20}$ The conclusion of the systematic review was that outreach nursing interventions improve disease-specific health-related quality of life.

However, some previous articles do not show exactly the same results. Rizzi et al. ${ }^{21}$ conducted a parallel cohort study and suggested that the particular home care program is effective in decreasing mortality. However, it should also be noted that the observing period of Rizzi et al. is 10 years, which is no more than 2 years in most of the studies included in this review. Therefore, the finding of this review indicates that more studies with an extended observation period are needed to prove the effect of home nursing support in mortality. On the other hand, Wong et al. also indicated that the effect on hospitalization was inconsistent, reducing admissions in one study but increasing them in others. ${ }^{20}$ Thus, in this respect, a conclusion about utilization of hospital services is still hard to draw. With the comparison, researchers studying mortality and utilization of hospital services with home nursing support intervention may be unable to reach a consistent 


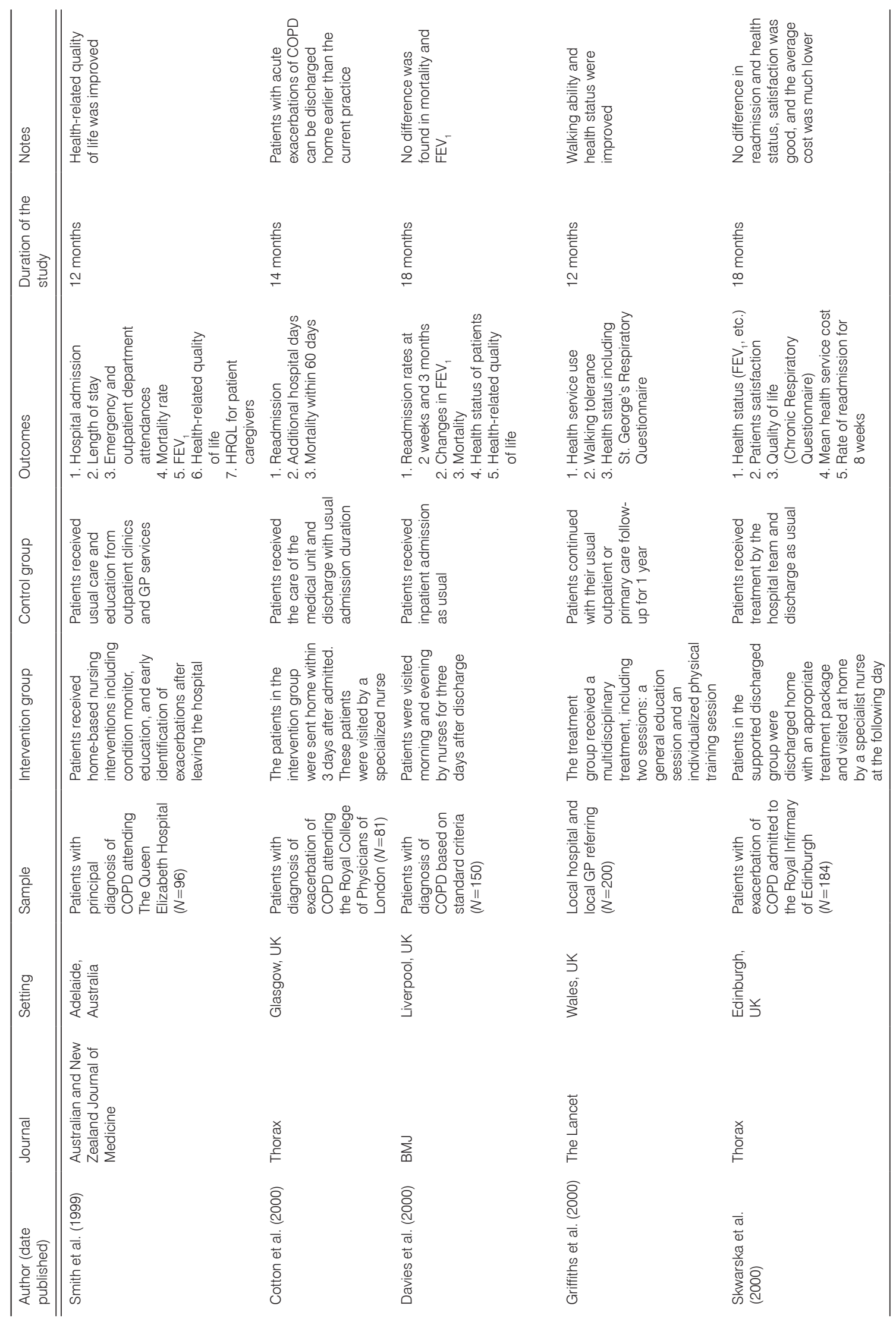

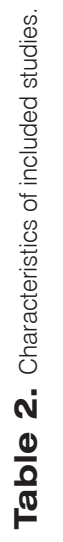




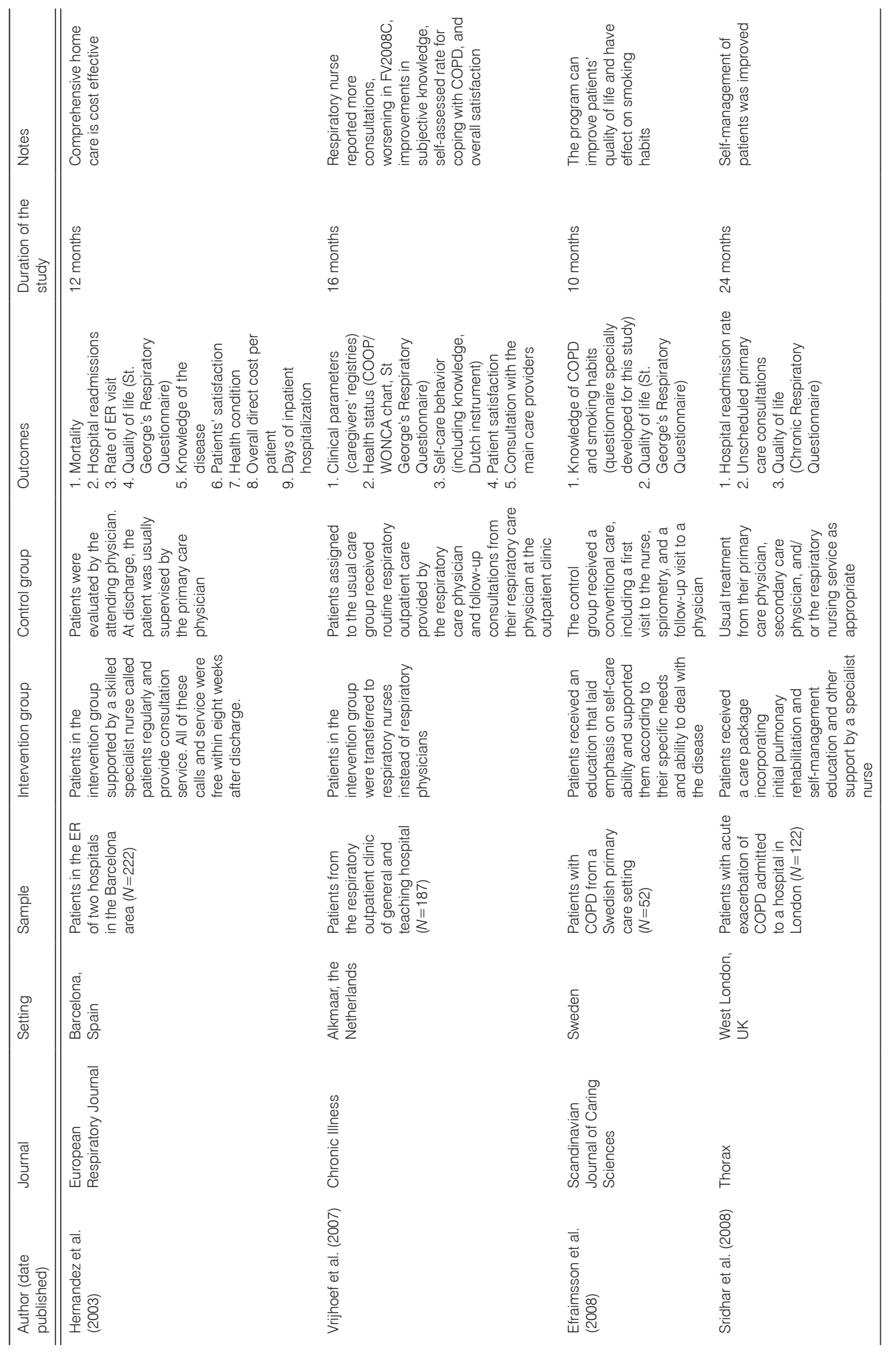

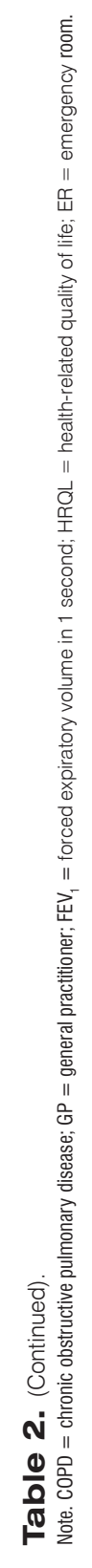



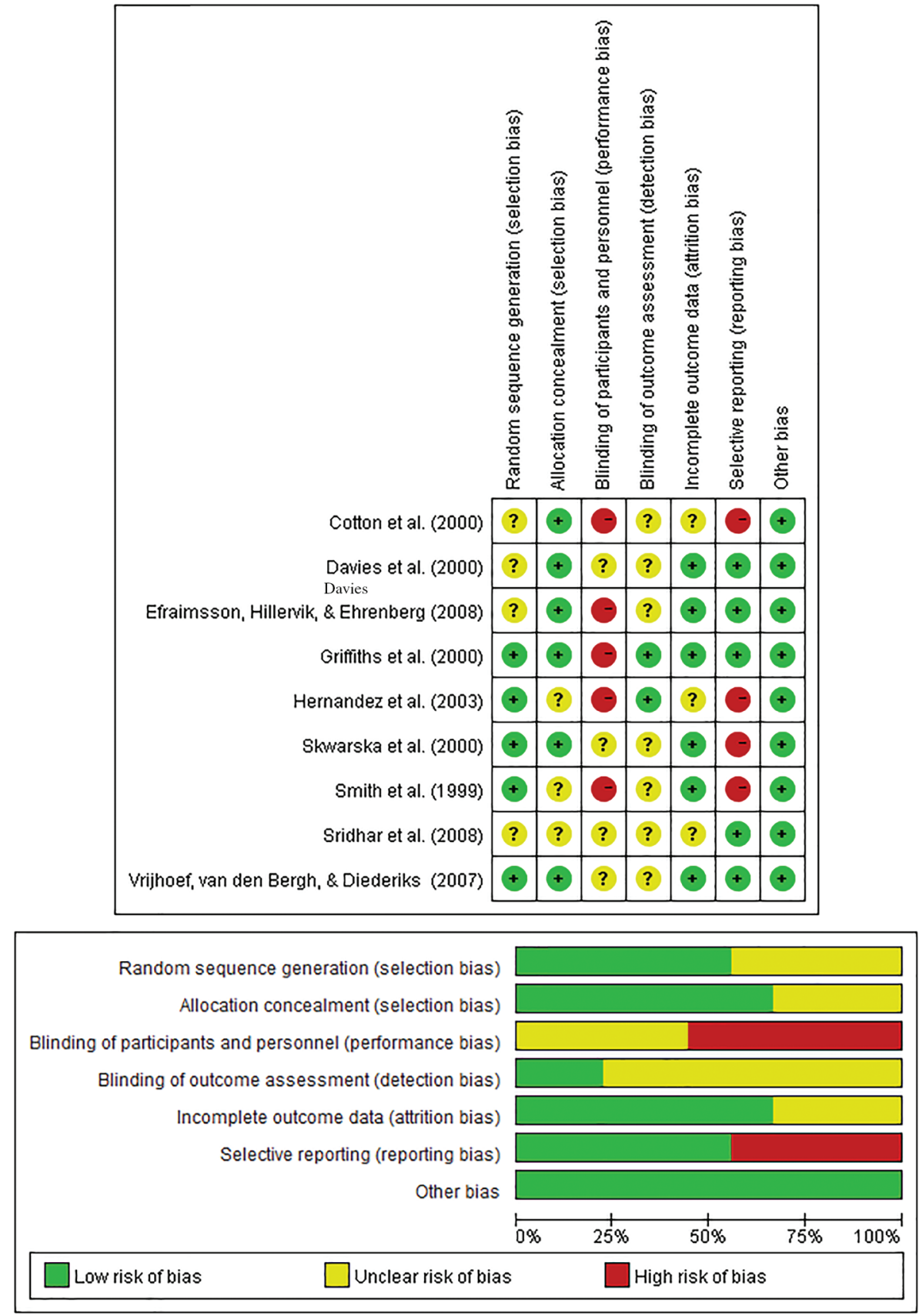

Figure 2. Summary of risk of bias for included trials.

conclusion at present. Although some findings require further development, the improvement of patients' quality of life is probably proven. This result may be positive for the current health facilities and policymakers.

\subsection{Supported discharge}

Cotton et al. and Skwarska et al. focused on the interventions by specialist nurses beginning with discharge.
In their studies, the utilization of hospital services, mortality, and lung function of patients may not show obvious improvement. However, these results are doubtful in one study, because there was no hypothesis test conducted between the intervention and control groups. The outcomes are only compared with the initial baseline. Therefore, the effectiveness of supported discharge in these aspects is not yet clearly proven. The cost of hospitalization is probably substantially decreased. 
This decrease indicates that intervention has an economic impact to some extent. At the same time, hospital readmission and patients' quality of life (specific in the chronic respiratory disease and patient satisfaction short postal questionnaires) have been almost proven to be equivalent between the intervention and control groups by the RCT of Skwarska et al.. These findings indicate that clinical specialist-supported discharge may be an alternative to the present admission process in some respects.

In the studies of both Cotton et al. and Skwarska et al., patients were sent home much earlier than usual and then supported by a nursing team at home. Thus, this intervention may release the beds in the hospital to other patients who need them. From this perspective, Barnett also suggested that supported discharge for patients with COPD can not only relieve stress on the medical bed management team, reducing long waits in the emergency room (ER) because no medical beds are available, but also provide patients with a quality service. ${ }^{22}$ Shepperd et al. also conducted a systematic review to determine the effectiveness of planning the discharge of patients. ${ }^{23}$ The results and conclusion prove that the structured discharge plan for individual patients probably reduces the length of hospital stay and readmission rates of patients. However, the impact of discharge planning on mortality, health outcomes, and cost remains uncertain. Compared to the review of Shepperd et al., this systematic review found a single study focusing on the cost of hospitalization. Although the mean health service cost significantly decreased in the intervention group in one included study, for the single evidence, we are cautious in drawing any conclusion about the economics of the service.

\subsection{Multidisciplinary cooperation program}

In the study of Griffiths et al., nurse specialists took part in a multidisciplinary cooperation program. ${ }^{14}$ Some individual improvements have been found in utilization of hospital services, but this finding is inadequate to draw a conclusion. On the other hand, an improvement in quality of life was found.

The multidisciplinary cooperation program or multidisciplinary team was developed in recent years. It is defined as an approach that increases the complexities of patient care, involving personnel from two or more disciplines with each discipline contributing its particular skill for the benefit of patients. ${ }^{24}$ With the development of the multidisciplinary team, nurse specialists' unique role becomes increasingly important in different disease fields, such as rheumatoid, urology, and many chronic diseases. ${ }^{25-27}$ As Wilkes et al. indicated, the community nurse in a multidisciplinary team for clients with chronic conditions has six main domains: advocate, supporter, coordinator, educator, team member, and assessor. ${ }^{28}$ Kruis and Chavannes ${ }^{29}$ and Zakrisson et al. ${ }^{30}$ conducted a 1-year longitudinal study with a quasi-experimental design in the multidisciplinary program with patients with COPD. They suggested that the multidisciplinary program in primary care produced a significant reduction in exacerbation frequency, but functional capacity and quality of life were unchanged.

However, the studies specifically on CNSs in a multidisciplinary team for COPD seem to be insufficient. The same problem appears in this review; the intervention involves CNSs, while the effectiveness is evaluated for the whole group. This limitation may be due to the particular characteristic of multidisciplinary team intervention.

\subsection{Nurse-led care for patients with COPD}

Sridhar et al. and Vrijhoef et al. conducted studies emphasizing the unique and significant role of CNSs. It is interesting to note that the results in numbers of caregiver consultations are inconsistent. Vrijhoef et al. reported more consultations in the intervention group. On the contrary, Sridhar et al. suggested that the numbers of consultations in the intervention group were obviously decreased. The reduction in consultations indicates many possibilities; maybe the patient's condition is stable or improving or maybe the patient does not care about the health problems anymore-in other words, the compliance worsens. ${ }^{31}$ Therefore, it is not reasonable to simply take fewer consultations as a "good" or "bad" trend; instead, we should examine the specific situations and consider patients' status and experiences. Unfortunately, these details in the studies of this review are not adequate. Thus, the finding regarding the numbers of consultations of medical personnel is an objectively reported paradox.

In some outcomes, such as hospital admission, mortality, patient's forced expiratory volume in 1 second, and several quality of life questionnaires, nurse- and physician-led services show no significant difference. Although the comparison could not lead to a conclusion that one is better than another, the lack of significant difference also indicates that nurse-led care service may be an alternative to doctor-led care service. Furthermore, CNSs provide some improvements for patients in the nurse-led care services. This finding is supported by many professionals. Carey suggested that generalpractice telephone triage and nurse clinics are two flexible approaches to delivering access to health care that is both quick and reliable. ${ }^{32}$ Cope et al. introduced that the nurse-led care service by respiratory CNSs led to 
improvement in both reducing the burden on the health care system and empowering patients to manage their conditions. ${ }^{33}$ Akinci and Olgun reported that a nurse-led home-based pulmonary rehabilitation program is conducted in Turkey. ${ }^{34}$ The result showed that this intervention brings meaningful improvements in patients' lung functional capacity and quality of life. From the existing evidence, the nurse-led care for patients with COPD may be a significant and positive attempt for the present health facilities and policies.

\subsection{Self-care management education}

Efraimsson et al. and Sridhar et al. explored the intervention in self-care education for patients with COPD. It is likely that one might believe that there are some improvements in mortality and quality of life while no positive results have been found in utilization of hospital services. Compared to our review, Monninkhof et al. conducted a systematic review on self-care management education of patients with COPD. ${ }^{35}$ This review systematically included eight studies of self-management education for patients with COPD compared to usual care. No improvement was found in hospital admissions, ER visits, days lost from work, and lung function of selfmanagement education. Two reasons may explain the differences in findings. First, the studies in the review of Monninkhof et al. were reported earlier than 2000 . The two articles in our review were published in 2008. With the development of self-care in these years, some improvements may have been made in the practice. On the other hand, as Monninkhof et al. indicated, the result of no effect on quality of life being detected may be because of the measurement instrument (HealthRelated Quality of Life Questionnaire). The two studies in our review involved three tools including St. George's Respiratory Questionnaire, Chronic Respiratory Disease Questionnaire, and Knowledge Questionnaire. These questionnaires may be more accurate and sensitive than the Health-Related Quality of Life Questionnaire for patients with COPD.

The nearest other studies, such as the study of Özkaptan and Kapucu, also suggested that the self-care model is effective in improving self-efficacy of patients with COPD. ${ }^{36}$ However, these studies may promote new concern regarding self-care management education. The objective, systematic review that is more comprehensive and high-quality is inadequate/required..

\section{Discussion}

This review aimed to examine the effectiveness of interventions by CNSs in patients with COPD. This review included nine RCTs and a total of five types of interventions.

\subsection{Evaluation of overall quality}

After comprehensive searching and screening, nine high-standard RCTs were retrieved in this review. According to Guyatt et al., the Grading of Recommendations, Assessment, Development and Evaluation (GRADE) approach was adopted to evaluate the quality of this review. ${ }^{37}$ As Higgins and Green explained, the GRADE approach states that "the quality of a body of evidence is the extent to which one can be confident that an estimate of effect or association is close to the quantity of specific interest." ${ }^{38}$ An RCT has a high-quality rating initially because of its design nature. Unfortunately, factors could affect the quality of evidence and downgrade RCT evidence to moderate, low, or even very low quality. These factors are limitations in the design and implementation of included studies, indicating a high likelihood of bias, indirectness of evidence, unexplained heterogeneity or inconsistency of results, imprecision of results, and high probability of publication bias.

\subsection{Limitation of the review}

This review has several limitations. First, due to the authors' limited language ability, only English and Chinese articles were retrieved. Studies in other languages may be missed. Second, although a comprehensive searching approach was conducted, most of the included study settings were in Europe and only one was in Australia. No studies in Asia or other regions were found. The setting of focus may also have led to some bias depending on different characteristics of ethnology. Finally, this review was conducted by only one person. As Higgins and Green indicated, a typical process should involve at least two investigators to select and evaluate studies independently. When the disagreements emerge in multiple authors, they may get final decisions with discussions. Unfortunately, this rigorous process happened in this review. Because of this limitation, all the studies were screened at least twice (generally five or six times) on different days.

\subsection{Implications for clinical practice and future research}

The findings of this review provide a series of evidence on the effectiveness of a CNS intervention for patients with COPD. On one hand, these findings may be a reference for the future nursing care of patients with COPD. On the other hand, the systematic review on the effects of CNSs' 
intervention for patients with COPD is insufficient. Therefore, this review may be a supplement for the unknown. Furthermore, by exploring the effectiveness of CNSs' intervention, the role of CNSs may help to define the direction of career development for some junior nurses. Although there are many studies on various interventions by CNSs, the high-quality evidence is insufficient. Except for some conflicting results, from which it is hard to draw a conclusion, more specific and rigorous studies are required. Owing to a lack of suitable evidence, a narrative synthesis was conducted. If more studies emerge in the future, it may be possible to carry out a meta-analysis on the effect of the CNS intervention for patients with COPD.

\section{Conclusions}

There is low- to moderate-quality evidence indicating that home nursing support interventions may have a

\section{References}

1. World Health Organization (WHO). Chronic obstructive pulmonary disease (COPD). 2015. http://www. who.int/respiratory/copd/en/. Accessed July 22, 2016.

2. Eisner MD, Anthonisen N, Coultas D, et al. An official American Thoracic Society public policy statement: Novel risk factors and the global burden of chronic obstructive pulmonary disease. Am J Respir Crit Care Med. 2010;182:693-718.

3. Morley JE. Chronic obstructive pulmonary disease: A disease of older persons. J Am Med Dir Assoc. 2014;15:151-153.

4. Donald F, Martin-Misener R, Carter N, et al. A systematic review of the effectiveness of advanced practice nurses in long-term care. $J$ Adv Nurs. 2013;69:2148-2161.

5. Rafferty S, Elborn S. The role of the respiratory nurse specialist. Clin Pulmonary Med. 2004;11:228-236.

6. French J, Bilton D, Campbell F. Nurse specialist care for bronchiectasis. Cochrane Database Syst Rev. 2003;3:CD004359.

7. Kuethe MC, Vaessen-Verberne AA, Elbers RG, Van Aalderen WM. Nurse versus physician-led care for the management of asthma. Cochrane Database Syst Rev. 2013;2:CD009296.

8. Rebar CR, Gersch CJ. Understanding Research for Evidence-Based Practice. Philadelphia, USA: Wolters Kluwer; 2015.

9. Davies L, Wilkinson M, Bonner S, Calverley PM, Angus RM. "Hospital at home» versus hospital care in patients with exacerbations of positive effect on mortality (low-quality evidence) and quality of life (moderate-quality evidence). There is moderate-quality evidence that no significant difference in quality of life has been found between the CNS-supported discharge intervention and the usual service. When CNSs participate in a multidisciplinary cooperation program, the effect of this intervention is evaluated as a whole. A moderate level of evidence has been found illustrating that this intervention probably has a positive effect on quality of life of patients with COPD. Both nurse-led care and self-care management education intervention likely have a positive effect on mortality of patients with COPD. This evidence is of moderate quality.

\section{Conflicts of interest}

All contributing authors declare no conflicts of interest. chronic obstructive pulmonary disease: Prospective randomised controlled trial. BMJ. 2000;321:1265-1268.

10. Hernandez C, Casas A, Escarrabill J, et al. Home hospitalisation of exacerbated chronic obstructive pulmonary disease patients. Eur Respir J. 2003;21:58-67.

11. Smith BJ, Appleton SL, Bennett PW, et al. The effect of a respiratory home nurse intervention in patients with chronic obstructive pulmonary disease (COPD). Aust N Z J Med. 1999;29:718-725.

12. Cotton MM, Bucknall CE, Dagg KD, et al. Early discharge for patients with exacerbations of chronic obstructive pulmonary disease: A randomized controlled trial. Thorax. 2000;55:902-906.

13. Skwarska E, Cohen G, Skwarski KM, et al. Randomized controlled trial of supported discharge in patients with exacerbations of chronic obstructive pulmonary disease. Thorax. 2000;55:907-912.

14. Griffiths TL, Burr ML, Campbell IA, et al. Results at 1 year of outpatient multidisciplinary pulmonary rehabilitation: A randomized controlled trial. Lancet. 2000;355:362-368.

15. Sridhar M, Taylor R, Dawson S, Roberts NJ, Partridge MR. A nurse led intermediate care package in patients who have been hospitalized with an acute exacerbation of chronic obstructive pulmonary disease. Thorax. 2008;63:194-200.

16. Vrijhoef HJ, Van Den Bergh JH, Diederiks JP, Weemhoff I, Spreeuwenberg C. Transfer of care for outpatients with stable chronic obstructive pul- 
monary disease from respiratory care physician to respiratory nurse - a randomized controlled study. Chronic IIIn. 2007;3:130-144.

17. Efraimsson EO, Hillervik C, Ehrenberg A. Effects of COPD self-care management education at a nurse-led primary health care clinic. Scand J Caring Sci. 2008;22:178-185.

18. Lee DT, Lee IF, Mackenzie AE, Ho RN. Effects of a care protocol on care outcomes in older nursing home patients with chronic obstructive pulmonary disease. $J$ Am Geriatr Soc. 2002;50:870-876.

19. Smith B, Appleton S, Adams R, Southcott A, Ruffin R. Home care by outreach nursing for chronic obstructive pulmonary disease. Cochrane Database Syst Rev. 2001;3:CD000994.

20. Wong CX, Carson KV, Smith BJ. Home care by outreach nursing for chronic obstructive pulmonary disease. Cochrane Database Syst Rev. 2012;4:CD000994.

21. Rizzi M, Grassi M, Pecis M, et al. A specific home care program improves the survival of patients with chronic obstructive pulmonary disease receiving long term oxygen therapy. Arch Phys Med Rehabil. 2009;90:395-401.

22. Barnett M. A nurse-led community scheme for managing patients with COPD. Prof Nurse. 2003;19:93-96.

23. Shepperd S, McClaran J, Phillips CO, et al. Discharge planning from hospital to home. Cochrane Database Syst Rev. 2010;1:CD000313.

24. Hall $P$, Weaver L. Interdisciplinary education and teamwork: A long and winding road. Med Educ. 2001;35:867-875.

25. Edwards K. Role of the clinical nurse specialist in the multidisciplinary team. Cancer Nurs Pract. 2011;10:22-26.

26. Lamb BW, Allchorne P, Sevdalis N, Vincent C, Green JSA. The role of the urology clinical nurse specialist in the multidisciplinary team meeting. Int J Urol Nurs. 2011;5:59-64.
27. Walker J. Rheumatoid arthritis: Role of the nurse and multidisciplinary team. $\mathrm{Br} J$ Nurs. $\overline{2012 ; 21: 334}, \overline{336-339 .}$

28. Wilkes L, Cioffi J, Cummings J, Warne B, Harrison K. Clients with chronic conditions: Community nurse role in a multidisciplinary team. J Clin Nurs. 2014; $23: \overline{84} 4-855$.

29. Kruis AL, Chavannes NH. A nurse-led multidisciplinary COPD programme: Potential flaws in the results? Prim Care Respir J. 2011;20:357; author reply 357-358.

30. Zakrisson AB, Engfeldt $P$, Hägglund $D$, et al. Nurseled multidisciplinary programme for patients with COPD in primary health care: A controlled trial. Prim Care Respir J. 2011;20:427-433.

31. Kunduru L, Kim SM, Heymen S, Whitehead WE. Factors that affect consultation and screening for fecal incontinence. Clin Gastroenterol Hepatol. 2015;13:709-716.

32. Carey N. The role of the minor illness nurse. Prim Health Care. 2005;15:37-40.

33. Cope K, Fowler L, Pogson Z. Developing a specialist-nurse-led 'COPD in-reach service'. $\mathrm{Br} J$ Nurs. 2015;24:441-445.

34. Akinci AC, Olgun N. The effectiveness of nurse-led, home-based pulmonary rehabilitation in patients with COPD in Turkey. Rehabil Nurs. 2011;36:159-165.

35. Monninkhof EM, Valk PDLPM, Palen J, et al. Selfmanagement education for chronic obstructive pulmonary disease. Cochrane Database Syst Rev. 2002;4:CD002990.

36. Bal Özkaptan B, Kapucu S. Home nursing care with the self-care model improves self-efficacy of patients with chronic obstructive pulmonary disease. Jpn J Nurs Sci. 2016;13:365-377.

37. Guyatt GH, Oxman AD, Kunz R, et al. GRADE guidelines: 8. Rating the quality of evidence indirectness. J Clin Epidemiol. 2011;64:1303-1310.

38. Higgins JP, Green S. Cochrane Handbook for Systematic Reviews of Interventions. Vol. 5. Chichester, UK: John Wiley \& Sons, Ltd.; 2008.

How to cite this article: Yin H, Yang L, Ye Q. A systematic review of the effectiveness of clinical nurse specialist interventions in patients with chronic obstructive pulmonary disease (COPD). Front Nurs. 2018; 2: $147-156$. https://doi.org/10.2478/fon-2018-0019 